\title{
Visual Search in Radial Menus
}

\author{
Krystian Samp and Stefan Decker \\ Digital Enterprise Research Institute, \\ National University of Ireland, Galway \\ \{krystian.samp, stefan. decker\}@deri.org
}

\begin{abstract}
Menu research has focused predominantly on linear menus (e.g., cascading menus). Little is known about user behavior with radial menus, which have been around for some time. The paper investigates the order in which users find items in radial menus. We analyze data collected in a controlled experiment and define serial position for items laid out in a circular fashion. For the first level (ring), the serial positions start at 12 o'clock position and alternate between both sides of the ring. For subsequent levels, the serial positions follow distance from a parent item. The defined search pattern yields strong fit and has substantial effect on search performance. We discuss the results in the context of radial menu design.
\end{abstract}

Keywords: Visual search, search patterns, radial menus, pie menus.

\section{Introduction}

Menus are a primary GUI technique and subject of considerable amount of research. One of the important areas of that research is how novice users search the menus. Although search strategies vary across the users [1], eye tracking studies demonstrate that search is predominantly top-to-bottom [4]. Consequently, top menu items are found faster (experiment 2 in [12]). This finding contributes to the understanding of user behavior with menus, the development of cognitive and menu performance models [5, 7], but also to improvements in menu design. For example, menu access can be improved by arranging items so the most important, frequently selected [14], recently selected [8], or user-specified [8] items are placed in the top of the menu for optimal search and selection.

Previous research focused predominantly on linear menus and similar findings regarding menu search are not available for radial menus. The findings regarding visual search in linear menus cannot be readily generalized to radial menus as the layout differences are considerable. Consequently, the question of how to arrange items in radial menus for optimal access remains unanswered. This question gains weight given the popularity of radial menus in research $[2,6,11,13]$, professional applications (e.g., Autodesk SketchBook Pro 2010), and computer games (e.g., Rockstar Red Dead Redemption 2010). The objective of this paper is to make the first step toward understanding how users search radial menus. 


\section{Method}

There are a number of plausible strategies for how the items on the rings could be searched - for example, clockwise, counter-clockwise, top-to-bottom or bottom-totop. The search could start from an item closest to the parent item or from an item on any end of an arc (see Figure 1, FAN and CRL-SMALL designs). Search might also lack any systematic patterns and be best described as random. Note, that the different search strategies result in different items being found first. Therefore, it is not clear how to arrange items for optimal menu access and it is necessary to analyze empirical data.

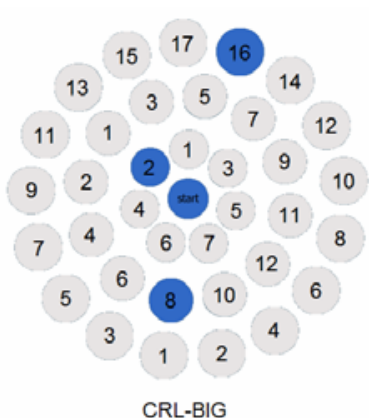

CRL-BIG

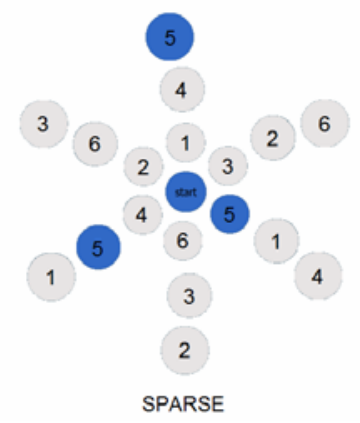

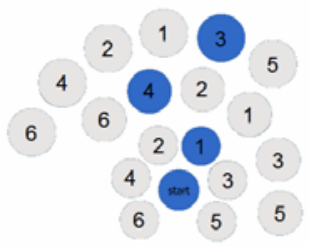

CRL-SMALL

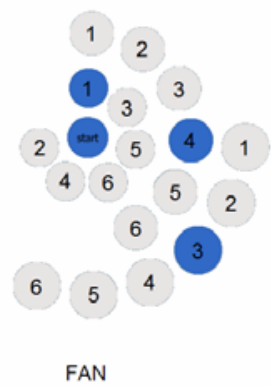

Fig. 1. Radial menus used in the experiment presented in [13]. The labels have been removed and substituted with serial positions. The selected items are colored blue.

We use the data collected in the experiment presented in [13]. In that experiment, 18 participants performed selections from one linear and four radial menus. The radial menus are presented in Figure 1.

The menus use concentric rings, one for each level, to organize the items. The most inner ring corresponds to the first level. The SPARSE design distributes items evenly within each ring. The FAN design places items clockwise around the ring starting from the top (for the first ring) or from the position closest to the parent item (for all subsequent rings). The Compact Radial Layout (CRL) design packs items tightly around their parent, keeping the distances between items and levels as short as 
possible. The behavior of the radial menus is similar to cascading menus. A user is initially presented only with the first level. A click on menu items post and un-post further levels (rings).

The menus used in the experiment had three levels and were of two different sizes: 1) small having 6 items on each level (CRL-SMALL, SPARSE and FAN in Figure 1); 2) big having 7, 12 and 17 items fully populating first, second and third level respectively (CRL-BIG in Figure 1). Menu content was randomly generated for each trial to prevent any learning effects and enforce visual search (i.e., to simulate novice behavior). A dictionary of nouns and adjectives was used to make the selection sequences easy to remember.

Visual search time was separated from pointing time using the Point-Completion Deadline (PCD) technique described in [10]. In short, the technique discourages participants from moving the mouse until they found the target. Consequently, the start of the mouse movement consistently marks the end of visual search. The PCD is an accepted, legit technique used to reliably separate and measure visual search times in selection tasks $[3,10,13]$. This technique, however, does not employ eye-tracking. Therefore, what we focus on in this paper is not patterns of the actual eye movements but rather patterns identifying order of finding the items. Such patterns would inform design of radial menus as to how order the items for optimal search, would shed some light at how users search radial menus, and would help formulate hypotheses for more detailed eye-tracking studies.

The decision to analyze the data from [13] was motivated by a number of reasons: 1) the designs presented in [13] resemble many radial menus used in production, such as those mentioned in the introduction; 2) the experiment in [13] included three different radial menus, two menu sizes, and three levels, enabling broader validation of our investigation; 3) the first levels of the designs in [13] are similar to the Pie Menu [6] in terms of searching; 4) the SPARSE design is similar to the Wave Menu 2 [3]. We hope these reasons will assure broader applicability of the results.

The authors in [13] reported average times for each menu design and each menu level. Therefore, from their study it is not known how position on the ring affects measured times or which items are found faster.

To find regularities in how participants search the rings, we aggregated visual search time across participants using an arbitrarily chosen but consistent scheme of numbering the items around the rings. This was done for each menu design and each menu level separately. Ordering the items according to the visual search times led to an observation that for the second and third levels, search time seemed to be a function of the distance from a parent item. For the FAN design this results in search following the ring clockwise starting from the item closest to the parent. For the CRLBIG, CRL-SMALL, and SPARSE designs the result is search alternating between both sides of the ring (i.e., half rings on both sides of the parent item), again starting from the item closest to the parent (consult with Figure 1). For the first level, where all the items are equally distant from the center, we observed that search starts from the top item (i.e., the item at 12 o'clock) and also alternates between both sides (in this case left and right) of the ring. 


\subsection{Results: Analyzing General Search Pattern}

Consistent with our observations, we define serial position of the items on the first ring in an alternating manner starting from the top item (i.e., the item at 12 o'clock). For all subsequent rings, serial position of the items is defined according to their distance from a parent item. Figure 1 superimposes serial positions on the items of the four radial menus. From now on, whenever we say the first or the last items, we refer to the order defined by the serial position.

We performed regression analysis to measure the actual relationship between the serial position and the visual search time. For each menu design and serial position, times were aggregated across participants and levels. It allowed us to obtain a number of observations necessary for establishing reliable mean time per serial position. Moreover, it allowed us to focus on the general pattern of which items are found faster regardless of menu level. If such a general pattern exists, it will be more readily applicable in practice compare to, for example, different patterns for different levels. We also performed a separate regression on times aggregated only across small radial menus.

Note that the aggregation across levels could flatten the possible effect of level, especially for the big radial menu, where each level contains a different number of items. To this end, we also analyze the effect of menu size later in the paper.

\subsubsection{Visual Search as a Function of Serial Position}

The average number of observations per serial position was 133 (SD 30) for small radial menus (CRL-SMALL, SPARSE, FAN) and 97 (SD 60) for big radial menu (CRL-BIG). The results are shown in Figure 2. The fit is strong across menu designs and two menu sizes $\left(\mathrm{R}^{2}>.85\right.$ and $\mathrm{R}^{2}=.99$ for times aggregated across three small radial menus). The results indicate that the defined search pattern holds for all menu designs used in the experiment. Interestingly, search alternating between both sides of the ring for the CRL-SMALL, CRL-BIG, and SPARSE designs (being a function of the distance from a parent item) leads to a non-optimal scanning path in terms of its total length. Search following circumference along one direction (e.g., clockwise starting in any position) would have resulted in a shorter path. We hypothesize that, not being able to find a target item in a given location, participants felt encouraged to move to an entirely different location.

Regression lines are fairly similar across small radial menus (intercepts ranging from $1044 \mathrm{~ms}$ to $1077 \mathrm{~ms}$ and slopes ranging from $61 \mathrm{~ms}$ to $76 \mathrm{~ms}$ ). Each subsequent serial position is associated with an average of $70 \mathrm{~ms}$ increase of visual search time. Regression line for the big radial menu has notably larger intercept (1410 ms) and slope $(100 \mathrm{~ms})$ when compared with the results for the small menus. For the big menu, each subsequent serial position is associated with an average of $100 \mathrm{~ms}$ increase of visual search time. It indicates that the menu size (length) affects search performance. We explore this in the next section.

Figure 2 shows that the difference between finding the first and the last item is substantial. For small radial menus, this difference is on average $360 \mathrm{~ms}$ (approximately $30 \%$ of the average visual search time for small menus). For the big radial menu the difference is $1900 \mathrm{~ms}$ (approximately $85 \%$ of the average visual search time for the big menu). Consequently, arranging items on the rings can lead to notable performance differences. 

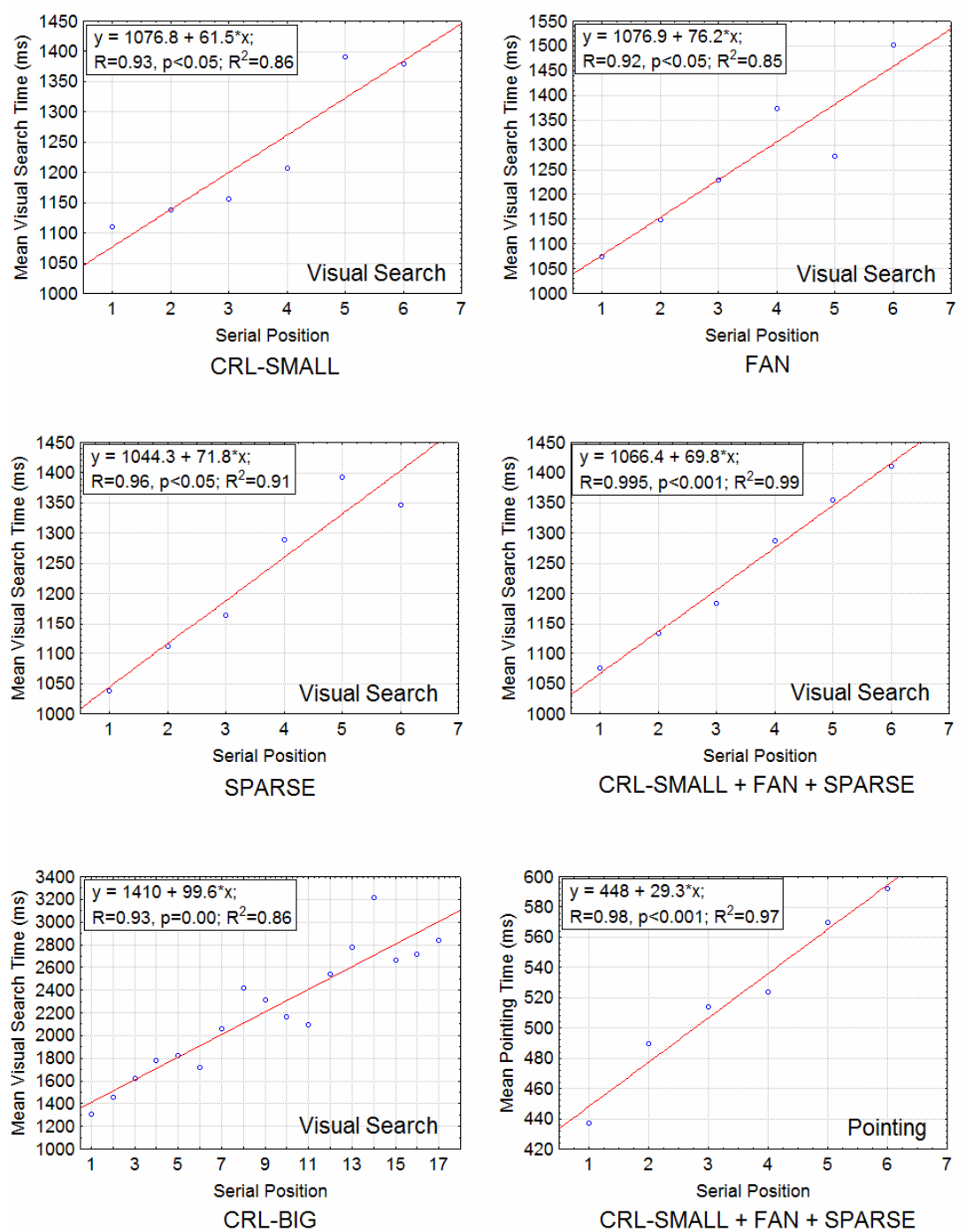

Fig. 2. Visual Search Time and Pointing Time as a function of serial position. The best-fit regression lines and corresponding statistics are provided for each condition. Notable dispersion of the visual search times after the seventh item for the CRL-BIG menu can be partially attributed to the smaller number of observations available for those item positions.

Note also that the defined serial position will also have linear effect on pointing time since each subsequent item (according to serial position) is more distant from a parent item and thus slower to select according to Fitts' law [9]. We run regression analysis of pointing times against serial position (see the last graph in Figure 2). As expected, the fit is strong $\left(\mathrm{R}^{2}=.97\right.$ for times aggregated across the three small radial menus, and $\mathrm{R}^{2}=.81$ for the big radial menu). Restricted by the available space, we did 
not include a graph for the big radial menu. Its lower correlation may be partially attributed to a smaller number of observations available for that menu. For small radial menus the difference between selecting the first and the last item is $160 \mathrm{~ms}$ (approximately $30 \%$ of the average pointing time for small menus). For the big radial menu, the difference is $300 \mathrm{~ms}$ (approximately $48 \%$ of the average pointing time for the big menu). Consequently, arranging items according to the proposed pattern (i.e., most important items in the first serial positions) not only will improve search time but also pointing to these items which is important for both, novices and experts [7].

\subsubsection{Visual Search as a Function of Menu Size}

The big radial menu (CRL-BIG) has different number of items on each level $(7,12$, 17). This allowed us to measure the effect of menu size on visual search time much like [12, 14] did for linear menus. We aggregated times for the seven first items on each level and performed regression between these times and the three menu sizes. Regression analysis produced a strong model with SearchTime $=140 *$ MenuSize + $209, \mathrm{R}^{2}=.99$. The visual search time increases by approximately $700 \mathrm{~ms}$ between the analyzed menu sizes ${ }^{1}$.

Menu size has similar linear effect on visual search time for radial menus as it has for linear menus. However, the size of the effect is notably larger for radial menus. Our results indicate that each additional item in radial menu increases average visual search time by $140 \mathrm{~ms}$. Cockburn et al. [7] report $80 \mathrm{~ms}$ increase for each additional item in linear menu. The difference is considerable. It would be interesting to explore which parameters of radial menus - for example, circular trajectory, item density, item spacing, item shapes - have the strongest effect on the slowdown.

\subsection{Results: Analyzing Individual Search Patterns}

Following our investigation, we analyzed individual search patterns. For each participant, menu design, and serial position, times were aggregated across the levels and plotted as a function of serial position (72 plots). We made two observations analyzing the individual plots. First, sometimes participants searched not one, but two or three consecutive items before moving to the opposite side of the ring. Second, participants employed not one but various search strategies, of which the following were dominant: 1) the search pattern described in the paper; 2) searching in an alternating manner but starting with the last items; 3) starting with the first items and searching half of the ring to the left/right before proceeding to the other half. Some searches could be best described as random. Note that for some of these strategies the items with the first serial positions were actually found the last ${ }^{2}$. For this reason, the pattern reported in our paper should not be treated as a definitive order in which participants find items on the ring but rather as general regularity (much like top-tobottom regularity in linear menus $[1,4]$ ). In other words, the pattern indicates which items are found faster and slower in a statistical sense - i.e., on average.

\footnotetext{
${ }^{1}$ We also performed the same analysis for four menu sizes (including size $=6$ from small menus). The results were almost identical, $\mathrm{R}^{2}=.98$.

${ }^{2}$ We tried alternative ordering schemas but they resulted in a poor fit. For practical reasons and space wise we focused on the prevailing search pattern.
} 
For the same reason, and to better understand strength of the proposed pattern, we decided to extend our analysis and check how often the first items are actually found faster. To this end, for each participant and menu design, we aggregated visual search time for each serial position. Further, for each participant and design, we counted how many items from the first half of all the items (according to our serial position) a participant found faster than the half of all the items (i.e., faster than the majority of the items). For each participant we average this count across the menus to obtain one count per participant - we call this count TOP-IN-TOP. Similarly, we counted how many items from the first half of all the items a participant found slower than the half of all the items. For each participant, the TOP-IN-BOTTOM is the average of this count across the menus. TOP-IN-TOP and TOP-IN-BOTTOM, divided by their sum, represent the probabilities of finding an item from the first half of the items faster/slower than a half of all the items (in other words faster/slower than the majority of the items). The distributions of TOP-IN-TOP and TOP-IN-BOTTOM measures were normal (the Shapiro-Wilk's W test used, $\mathrm{p}>0.05$ ). We performed the dependent measures t-test to determine if TOP-IN-TOP and TOP-IN-BOTTOM differed significantly.

For small radial menus, the mean number of TOP-IN-TOP items was 2 (SD 0.58) (associated probability 67\%) and the mean number of TOP-IN-BOTTOM items was 1 (SD 0.58) (probability 33\%). For the big radial menu, the mean number of TOP-INTOP items was 6.5 (SD 1) (probability 72\%) and the mean number of TOP-INBOTTOM items was 2.5 (SD 1) (probability 28\%).

The results of the t-test show that, indeed, the first half of items determined by our serial position are found faster than the majority of items $(\mathrm{p}<.05$ for small radial menus, and $\mathrm{p}<.01$ for the big radial menu). This reinforces the results obtained in the regression analysis. However, reported means and probabilities demonstrate that the search pattern determined by the serial position is not as strong as could be asserted solely on the basis of correlation score. In other words, other search patterns are also important

In sum, placing the most important items according to the proposed pattern gives approximately $70 \%$ of chances for these items to be found faster than the majority of the items. This is $20 \%$ better than a random order. Given substantial differences between visual search time for the items found the fastest and the slowest $(30-85 \%$, see section 2.1.1), following the proposed pattern will lead to better overall performance of radial menus. This is important as searching radial menus is known to be slower than searching linear menus [13].

The existence of other patterns hints that designers might consider solutions that suggest the sense of reading, such as using an arrow or using animation which reveals the items in a desired order.

\section{Conclusions}

In this paper we investigated the order in which the items are found in radial menus. We found that for the second and third levels, search time is a function of the distance from a parent item. For the first ring, where all the items are equally distant from the center, search alternates between both sides of the ring, starting from the top item. 
The difference between finding the first and the last item is substantial: $30 \%$ and $85 \%$ of the average search time in small and big radial menu respectively. Given that the search is a dominant component of menu selection times for novices [7], the order of the items on a ring will affect menu performance.

Our results suggest that the items a designer wishes to be found faster (e.g., most important items) should be placed in the top of the first ring and in the positions closest to the parent item for all subsequent levels. Users will also benefit from decreased pointing times to these items.

The future work could use eye-tracking devices to explore the actual eye movement patterns.

\section{References}

1. Aaltonen, A.: Hyrskykari, and A., Räihä, K. J.: 101 spots, or how do users read menus? In: International Conference on Human Factors in Computing Systems (CHI), pp. 132-139 (1998)

2. Bailly, G.: Wave Menus: Improving the novice mode of Marking Menus. INTERACT, 475-488 (2007)

3. Burke, M., Hornof, A.J., Nilsen, E., Gorman, N.: High-cost banner blindness: Ads increase perceived workload, hinder visual search, and are forgotten. ACM ToCHI 12(4) (2005)

4. Byrne, M., Anderson, J., Douglass, S., Matessa, M.: Eye Tracking the Visual Search of Click-Down Menus. In: CHI, pp. 402-409 (1997)

5. Byrne, M.D.: ACT-R/PM and menu selection: applying a cognitive architecture to HCI. International Journal Human-Computer Studies 55, 41-84 (2001)

6. Callahan, J., Hopkins, D., Weiser, M., Shneiderman, B.: An empirical comparison of pie vs. linear menus. In: CHI, pp. 95-100 (1988)

7. Cockburn, A., Gutwin, C., Greenberg, S.: A Predictive Model of Menu Performance. In: CHI, pp. 627-636 (2007)

8. Findlater, L., McGrenere, J.: A comparison of static, adaptive, and adaptable menus. In: CHI, pp. 89-96 (2004)

9. Fitts, P.: The Information Capacity of the Human Motor System in Controlling the Amplitude of Movement. Journal Experimental Psychology 47, 381-391 (1954)

10. Hornof, A.J.: Visual Search and Mouse-Pointing in Labeled versus Unlabeled TwoDimensional Visual Hierarchies. ACM ToCHI 8(3), 171-197 (2001)

11. Kurtenbach, G.: The Design and Evaluation of Marking Menus. PhD Thesis (1993)

12. Nilsen, E.L.: Perceptual-motor control in human-computer interaction (Tech. Rep. No. 37). Ann Arbor, Michigan: The Cognitive Science and Machine Intelligence Laboratory, The University of Michigan (1991)

13. Samp, K., Decker, S.: Supporting menu design with radial layouts. In: International Conference on Advanced Visual Interfaces (AVI), pp. 155-162 (2010)

14. Sears, A., Shneiderman, B.: Split menus: effectively using selection frequency to organize menus. ACM ToCHI 1(1), 27-51 (1994) 